\title{
Pd-Mediated Cross-Coupling Reactions between the Bromide of Baylis-Hillman Adduct and Organostannanes
}

\author{
Saravanan Gowrisankar, Sung Hwan Kim, and Jae Nyoung Kim* \\ Department of Chemistry and Institute of Basic Science, Chonnam National Cniversity, Gwangiu 500-757, Korea \\ E-mail: kiminachonnamac.kr \\ Recerved September 20, 2008, Accepted January 14, 2009
}

Key Wonds: Palladium, Cross-coupling, Baylis-Hillman adduct. Organostannanes

Recently numerous chemical transformations of BaylisHillman adducts have been published involving the synthesis of cyclic and acyclic compounds. Among the reactions introduction of allyl ${ }^{2}$ vinyl $1^{3}$ and ary $1^{4.5}$ moiety at the primary position of the Baylis-Hillman adducts can be regarded as an important transformation due to the usefulness of the compounds thereof as synthetic intermediates. ${ }^{2-5}$

Introduction of allyl group at the primary position of BaylisHillman adduct was studied by Yaday and coworkers. ${ }^{\text {Zab }}$ They reported the syththesis of 1.5 -diene derivatives involving the use of $i n$-situ generated ally lzine reagent and Baylis-Hillman acetates. ${ }^{\text {a }}$ Later. they reported another method. the reaction of allyltrimethylsilane and Baylis-Hillman alcohol in the presence of $\mathrm{BF}_{3}$ etherate. ${ }^{\mathrm{z}}$ Introduction of alkenyl moiety at the primary position of Baỵlis-Hillman adduct has not been reported much. ${ }^{3}$ Isobutenyl and isopentenyl groups were introduced using the corresponding Grignard reagents and used for the sytnthesis of dihydronaphthalenes. ${ }^{3 a}$ Very recently Ranu and coworkers published an elegant method. a Pd-mediated introduction of aryl and vinỵl moiety to Baỵlis-Hillman acetate. using triorganoindium reagents. ${ }^{\text {bb }}$ Introduction of aryl group at the primary position of the Baylis-Hillman adduct has been studied relatively well and carried out by using Friedel-Crafts type reaction under various acidic conditions. ${ }^{4}$ However. regioselectivity is the principle problem in this case. ${ }^{+6,1 !}$ As an example. Friedel-Crafts reaction with toluene produced a mixture of ortho- and para-isomers (vide infra, Scheme 2). In addition, various $\mathrm{Pd}-$ and $\mathrm{Rh}$-catalyzed introduction of aryl moiety has been studied. Kabalka group reported a Pd-catalyzed cross-coupling of Baylis-Hillntan acetate and organosilane $^{\text {5a }}$ or potassium organotrifluoroborate ${ }^{\text {st }}$ Genet and coworkers examined a Rlh-catalyzed arylation with arylboronic acid ${ }^{s_{c}}$ or potassium aryltrifluoroborate ${ }^{\text {sd }}$

Although various methods have been reported and most of the methods provided moderate yields of products. ${ }^{2.5}$ development of an efficient and general method for the introduction of carbon nucleophile at the primary position of Baylis-Hillntan adduct is still highly required. especially in a stereoselective manner. Thus we decided to examine the cross-coupling reactions between the bromide of Baylis-Hillman adduct and allyl-, anyl-, and vinylstannane (Scheme 1) as a continuous work on our recent Pd-mediated reactions with Baylis-Hillman adducts. ${ }^{6}$ Although cross-couplings involving the use of allyl bromides and organostannanes have been studied extensively. "this is the first trial in the Baylis-Hillman chemistry to the best of our knowledge. Fortunately we obtained good results and wish to report herein the results. The results are summarized in Scheme 1 .

We decided to use the bromides of Baylis-Hillman adducts and chose three representative Baylis-Hillman adducts. 2a-c. ${ }^{8}$ The starting materials $\mathbf{2 a - c}$ were prepared in pure form ( $Z$ for $\mathbf{2} \mathbf{a}$ and $\mathbf{2 b} . E$ for $\mathbf{2 c}$ ) as shown in Scheme $1 .{ }^{9.10}$ The reaction of cimnamyl bromide $\mathbf{2} \mathbf{a}$ and ally ltributy'lstannane ( 1.2 equiv) in the presence of $\mathrm{Pd}\left(\mathrm{Ph}_{3} \mathrm{P}\right)_{4} / \mathrm{LiCl}(0.5$ equiv) in THF afforded desired product $3 \mathrm{a}$ in $96 \%$ yield ${ }^{\left.\hat{a}_{0}(L)\right]}$ As a palladium catalyst, $\mathrm{Pd}\left(\mathrm{Pl}_{3} \mathrm{P}\right)_{4}$ was superior than $\mathrm{Pd}(\mathrm{OAc})_{2}$ and only 2

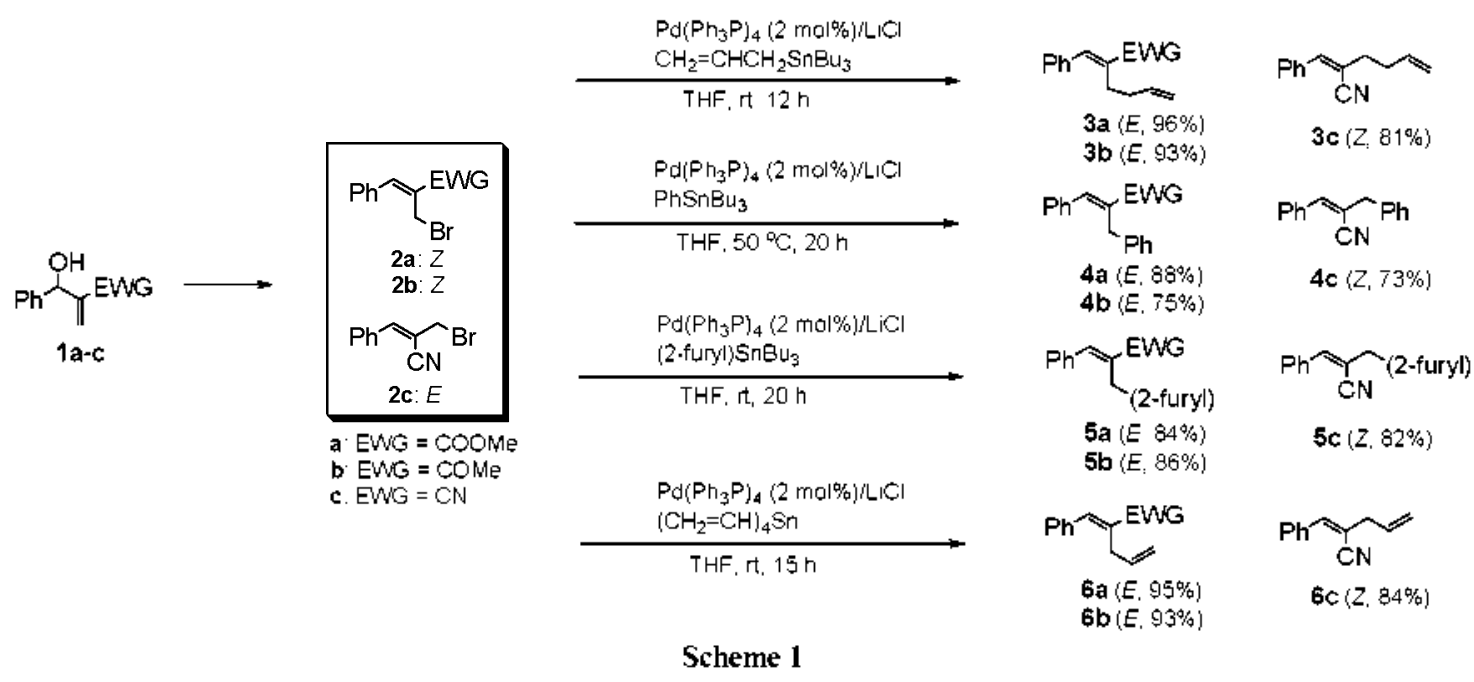


<smiles>COC(=O)/C=C\c1ccc(C)cc1</smiles>
$4 d(78 \%)$

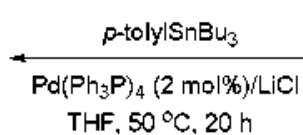

THF, $50^{\circ} \mathrm{C}, 20 \mathrm{~h}$
$2 a$

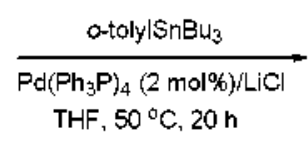<smiles>COC(=O)/C=C(\Cc1ccccc1)c1ccccc1</smiles>
$4 d+4 e(74 \%, 45: 55)$

$1 \mathrm{a}$

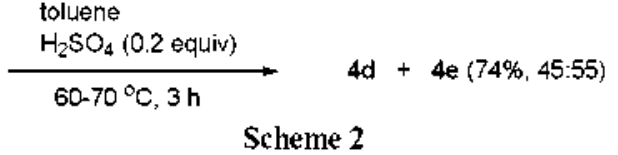

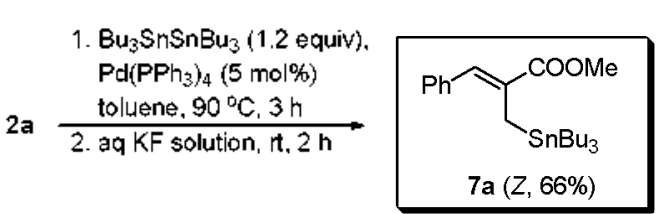

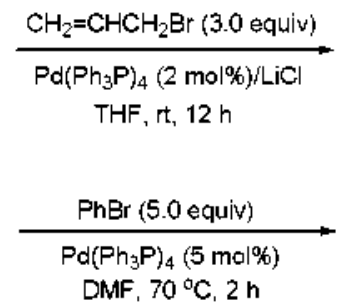

DMF, $70^{\circ} \mathrm{C}, 2 \mathrm{~h}$

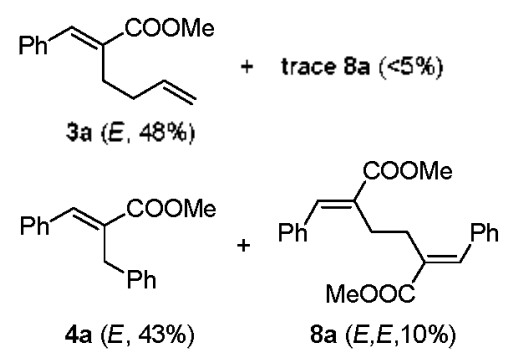

$4 a(E, 43 \%)$

Scheme 3

mol $\%$ of catalyst was sufficient. The reaction time can be shortened by raising the reaction temperature with $5 \mathrm{~mol} \%$ of $\mathrm{Pd}(0)$ catalyst. Similarly, 3b and $3 \mathbf{c}$ were prepared in high yields also $(81-93 \%)$. The arylation and viny lation of 2 a-c were examined with phenyltributỵlstannane. 2-(tributỵlstannyl)furan. and tetravinylstannane. The corresponding products $4 \mathbf{a}-\mathbf{c}$. 5a-c, and 6 a-c were obtained in good yields $(73-95 \%)$. The yields of nitrile-containing compounds were comparatively lower than those of the ester- or acetyl-containing compounds. The reaction of phenyltributylstannane required elevated temperature $\left(50^{\circ} \mathrm{C}\right)$.

Tolyl-substituted products $4 \mathbf{d}$ and te were synthesized in good yields (74-78\%) similarly by the reactions of $\mathbf{2 a}$ and ortho-tolyltributylstannane and para-tolyltributylstanuane which were prepared from Pd-mediated reactions between n-tributyltin hydride and 2-iodo- and 4-iodotoluene. respectively while the traditional Friedel-Crafts reaction of Baylis-Hillman adduct $\mathbf{1 a}$ and toluene under the influence of $\mathrm{H}_{2} \mathrm{SO}_{4}(0.2$ equiv) afforded ortho para mixture in $74 \%$ yield (55:45 ratio in ${ }^{\mathrm{H}} \mathrm{H} \mathrm{NMR}$ ) as in Scheme 2.

As a next trial we prepared tributylstanyl derivative 7 a by the Pd-mediated reaction of $\mathbf{2 a}$ and hexabutylditin in the presence of $\mathrm{Pd}(0)$ in $66 \%$ yield as in Scheme $3 .^{13.14}$ The reaction of $7 \mathbf{a}$ and allyl bromide under the influence of $\mathrm{Pd}\left(\mathrm{Ph}_{3} \mathrm{P}\right)_{4} / \mathrm{LiCl}$ produced $3 \mathrm{a}$ in low y ield $(48 \%)$. Similarly the reaction of $7 \mathrm{a}$ and bromobenzene afforded $4 \mathrm{a}$ in low yield $(43 \%)$ also together $w$ ith some self-coupling product $\mathbf{8 a}$ $(10 \%)^{15}$

In summary. we disclosed an efficient synthetic method of ally l- aryl-and vinyl-attached Bay lis-Hillman adducts wio the Pd-mediated cross-coupling reactions between the bromide of Baylis-Hillman adduct and the corresponding organostannane compounds.

\section{Experimental Section}

Typical experimental procedure for the preparation of $\mathbf{3 a}$.
To a stirred mixture of $2 \mathrm{a}^{10}$ (128 $\mathrm{mg} .0 .5 \mathrm{mmol}$ ) and allyltributylstannane (200 ntg, $0.6 \mathrm{mumol})$ in dry THF (2 nL) was added $\mathrm{Pd}\left(\mathrm{PPh}_{2}\right)_{4}(12 \mathrm{mg} .2 \mathrm{~mol} \%)$ and $\mathrm{LiCl}(\mathrm{ll} \mathrm{mg} .0 .25$ nimol) and the reaction mixture was stirred at room temperature for $12 \mathrm{~h}$. After removal of solvent and column chromatographic purification process (hexanes/EtOAc. 95:5) analytically pure product 3 a was obtained as colorless oil. ${ }^{\text {?a }}$ $104 \mathrm{mg}(96 \%)$. Other compounds were sy nthesized similarly and the known compounds, $3 a^{2 a}+a^{5 b}+b^{5 a}+c^{5 e}+d^{5 b}+e^{5 b}$ $6 a^{36} 6 c^{16}$ and $8 a^{15}$ were identified by comparison with their ${ }^{1} \mathrm{H}$ NMR and IR spectroscopic data with the reported. The spectroscopic data of unknown compounds, 3b. 3c. 5a 5 b. 5c. $6 \mathbf{b}$ and $7 \mathbf{a}$. are as follows.

Compound 3b: 93\%; colorless oil; IR (film) 3077, 2927. $1667 \mathrm{~cm}^{-1}$ : ${ }^{1} \mathrm{H}$ NMR (CDCl $\left.3.300 \mathrm{MHz}\right) \delta 2.20-2.24$ (m. $2 \mathrm{H}$ ), $2.45(\mathrm{~s}, 3 \mathrm{H}), 2.60-2.63(\mathrm{~m}, 2 \mathrm{H}), 4.95-5.05(\mathrm{~m}, 2 \mathrm{H}) .5 .78-5.87$ (m. IH). 7.34-7.43 (m, 5H). 7.5l (s. IH): ${ }^{13} \mathrm{C} \mathrm{NMR} \mathrm{(CDCl}$. $75 \mathrm{MHz})$ ò 25.61, 26.07, 33.00. 114.77, 128.56, 128.60 . 129.20. 135.57. 137.88.140.02, 1+2.06, 200.06 .

Compound 3c: $81 \%$ : colorless oil: IR (film) 3080. 2924. $2209 \mathrm{~cm}^{.1}$ : ${ }^{1} \mathrm{H} \mathrm{NMR}\left(\mathrm{CDCl}_{3} .300 \mathrm{MHz}\right) \delta 2.37-2.54$ (n. $\left.4 \mathrm{H}\right)$, $5.04-5.16(\mathrm{~m} .2 \mathrm{H}) .5 .75-5.88(\mathrm{~m} . \mathrm{lH}) .6 .94$ (s. lH). 7.26-7.44 (m. 3H), 7.69-7.74 (m, 2H): ${ }^{13} \mathrm{C} \mathrm{NMR}\left(\mathrm{CDCl}_{2} .75 \mathrm{MHz}\right) \delta$ $32.27,35.64 .110 .66,116.45,118.65,128.55$. 128.78. 129.93, 133.67. 136.04. 143.84

Compound 5a: $84 \%$, yellow oil; IR (film) $2951,1715 \mathrm{~cm}^{-1}$; ${ }^{1} \mathrm{H}$ NMR (CDCl $\left.3,300 \mathrm{MHz}\right)$ ò 3.80 (s. $\left.3 \mathrm{H}\right) .3 .88$ (s. $2 \mathrm{H}$ ), $6.06-6.08(\mathrm{~m}, 1 \mathrm{H}) .6 .30-6.31(\mathrm{~m}, 1 \mathrm{H}), 7.33-7.47(\mathrm{~m}, 6 \mathrm{H})$, 7.87 (s. IH): ${ }^{13} \mathrm{C} \mathrm{NMR}\left(\mathrm{CDCl}_{3} .75 \mathrm{MHz}\right)$ ò 26.89. 52.15 . 106.00. 110.39. 129.23. 128.55. 128.89. 129.33, 134.99. $1+1.23 .1+1.30 .152 .96 .168 .22$.

Compound 5b: 86\%: yellow oil: IR (film) 2957, 2924. 1669 $\mathrm{cm}^{-1} ;{ }^{\mathrm{H}} \mathrm{H} \mathrm{NMR}\left(\mathrm{CDCl}_{3}, 300 \mathrm{MHz}\right) \delta 2.46$ (s. $\left.3 \mathrm{H}\right), 3.87$ (s. $2 \mathrm{H}$ ). $6.00-6.02(\mathrm{~m}, \mathrm{lH}) .6 .27-6.29(\mathrm{~m}, \mathrm{lH}), 7.32-7.49(\mathrm{~m}, 6 \mathrm{H})$. 7.68 (s. $1 \mathrm{H}) ;{ }^{13} \mathrm{C}$ NMR (CDCl $\left.2.75 \mathrm{MHz}\right)$ ò 25.78, 26.05, 106.01. 110.37. 128.60. 129.04. 129.36. 134.99, 137.45, 141.14. 141.45. 153.04. 199.13. 
Compound 5c: $82 \%$; yellow oil; IR (film) $2913.2212 \mathrm{~cm}^{-1}$ : ${ }^{1} \mathrm{H}$ NMR $\left(\mathrm{CDCl}_{3} .500 \mathrm{MHz}\right)$ ò 3.73 (s. $\left.2 \mathrm{H}\right), 6.26-6.27(\mathrm{~m}$. 1H). 6.35-6.36 (m. 1H). $6.99(\mathrm{~s}, 1 \mathrm{H}), 7.38-7.43(\mathrm{~m}, 5 \mathrm{H})$. 7.72-7.74 (m. 2H); ${ }^{13} \mathrm{C} \mathrm{NMR}\left(\mathrm{CDCl}_{3}, 75 \mathrm{MHz}\right) \delta 34.58$. $107.60 .108 .00,110.63 .118 .33,128.73,128.80,130.28$. 133.34. 142.46, 144.84, 149.82.

Compound 6b: 93\%: colorless oil: $\mathbb{R}$ (film) 3079. 2978. $1668 \mathrm{~cm}^{-1}$ : ${ }^{1} \mathrm{H}$ NMR $\left(\mathrm{CDCl}_{3}, 300 \mathrm{MHz}\right)$ ò 2.47 (5, 3H). 3.26-3.29 (m. 2H). 4.99-5.11 (m. 2H). 5.91-6.03 (m. IH). $7.32-7.46(\mathrm{~m} .5 \mathrm{H}), 7.64$ (s. IH): ${ }^{13} \mathrm{C} \mathrm{NMR}\left(\mathrm{CDCl}_{3}, 75 \mathrm{MHz}\right)$ ò $26.05,30.57,115.51 .128 .48,128.82,129.28,135.34$. 135.73. 139.20, 141.03. 199.54

Compound 7a: 66\%; colorless oil; IR (film) 2956, 2925. $1709 \mathrm{~cm}^{-1}:{ }^{1} \mathrm{H} \mathrm{NMR}\left(\mathrm{CDCl}_{3}, 300 \mathrm{MHz}\right) \hat{o} 0.84-0.94(\mathrm{~m}, 15 \mathrm{H})$. $1.23-1.31(\mathrm{~m}, 6 \mathrm{H}), 1.37-1.46(\mathrm{~m} .6 \mathrm{H}) .2 .29$ (s. 2H). 3.80 (s.

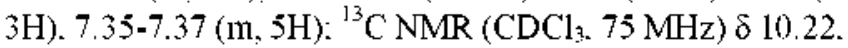
$11.80,13.67,27.33,28.95,51.98,127.47,128.30,129.25$. 131.78. 133.89, 136.88, 169.46: ESIMS $m / z+65\left(\mathrm{M}^{+}+1\right)$. Anal. Calcd for $\mathrm{C}_{23} \mathrm{H}_{38} \mathrm{O}_{2} \mathrm{Sn}:$ C. 59.37: H. 8.23. Found: C. 59.02: H, 8.44.

Acknowledgments. This work was supported by the Korea Research Foundation Grant funded by the Korean Govemment (MOEHRD. KRF-2007-313-C00417). Spectroscopic data was obtained from the Korea Basic Science Institute, Gwangju branch.

\section{References and Notes}

1. For general review on Baylis-Hillman reaction, see: (a) Basavaial, D.: Rao, A. J.: Satyanarayana, T. Chem. Rev. 2003, 103 . 811-891. (b) Ciganek, E. In Organic Reactions; Paquette, L. A., Ed.: Toln Wiley \& Sons: New York, 1997; Vol. 51, pp 201-350. (c) Basavaiah, D.; Rao, P. D.: Hyma, R. S. Tetrahedron 1996, 52 , 8001-8062. (d) Kim. J. N.; Lee, K. Y. Cur. Org. Chent 2002, 6, 627-645. (e) Lee, K. Y.; Gownsankar, S.: Kim, J. N. Bull. Korean Chem. Soc 2005, 26, 1481-1490. (f) Singh, V: Batra, S. Tetwhedron $2008,64,4511-4574$ and further references cited therein.

2. For the synthesis of ally lated Bay lis-Hillman adducts, see: (a) Srihari, P.: Singh, A. P.: Basak, A. K.; Yadav, T. S. Tetrahedron Lett. 2007. 18, 5999-6001. (b) Yadav. I. S.: Reddy, B. V. S.: Mandal, S. S. Singh. A. P. Basak, A. K. Sinthesis 2008, 1943-1947. (c) Yadav, T. S.: Reddy, B. V. S.: Singh. A. P.: Basak. A. K. Snthesis 2008. 469-473. (d) Mandal. S. K.: Paira, M: Rov, S. C. J. Ory. Chent 2008, 73, 3823-3827.

3. For the synthesis of vinyl moiety-containing Baylis-Hillman adducts, see: (a) Gowrisankar, S.: Lee, C. G.: Kim, J. N. Tetrahedron Lett. 2004, 45, 6949-6953. (b) Ranin, B. C.: Chattopadhyay, K.: Tan1a. R. Tetrahedron Lett. 2007, $48,3847-3850$.

4. For the sunthesis of arylated Bavlis-Hillman adducts by Friedel-Crafts reaction, see: (a) Basavaiah, D.: Pandiaraju, S: Padmaja, K. Sinlen 1996, 393-395. (b) Basavaiah, D.; Krishnamacharyulu. M.: Hyma, R. S.: Pandiaraju. S. Tetrahedron Lett. 1997, 38, 2141-2144. (c) Lee, H. J.: Seong, M. R.: Kinn. T. N. Tetrahedron Lett. 1998, 39, 6223-6226. (d) Das, B.; Majhi. A.; Banerje, I: Chowdhury. N.: Venkateswarlu. K. Chem. Lett. 2005, 3t, 1492-1493. (e) Shanmugam, P.; Rajasingh, P. Chent. Lett. 2005, 34, 1494-1495.

5. For the synthesis of ary lated Bay lis-Hillman adducts using metal catalyst see: (a) Kabalka, G. W.: Dong, G.; Venkataiah, B.; Chen, C. J. Org. Chem. 2005, 70, 9207-9210, (b) Kabalka, G. W. Venkataiah, B.: Dong, G. Org. Left. 2003, 5, 3803-3805. (c) Navarre, L.: Darses, S.; Genet, J.P. Chent Conmmin. 2004, 1108-1109. (d) Navarre, L.: Darses, S.: Genet. J.-P. Atw Swth.
Catal. 2006, 348, 317-322. (e) Kantam. M. L.: Kumar, K. B. S.; Sreedhar, B. J. Oig. Chem, 2008, 73, 320-322.

6 . For our recent contributions on $\mathrm{Pd}$-mediated reactions of modified Bay.lis-Hillman adducts, see: (a) Gowrisarkar, S.; Lee, H. S.: Kim. J. M: Kim. I. N. Tetrahedron Lett. 2008. 49. 1670-1673. (b) Kim, I. M.: Kim, K. H.: Kim, T. H.; Kim, I. N. Tetrahedron Lett. 2008, 49, 3248-3251. (c) Gowrisankar. S: Lee, H. S.; Lee, K. Y.; Lee, T.-E.; Kim, T. N. Tetwhedron Lent. 2007, 48, 8619-8622. (d) Lee, H. S.; Kim, S. H.: Kim. T. H.: Kim, J. N. Tetrahedron Lett. 2008, 49, 1773-1776. (e) Kim, H. S.; Gowrisankar, S.: Kim, S. H.: Kim, J. N. Tetrahedron Lett. 2008. 19, 3858-3861. (f) Lee, H. S.: Kim, S. H.; Gowrisankar, S.; Kim, J. N. Tetrahedron 2008, 64, 7183-7190

7. For the Pd-mediated cross-coupling reactions between ally lic bromides (and acetates) and organostannanes. see: (a) Godschal., I, Stille, J. K. Tetrahedron Lett. 1980, 21, 2599-2602. (b) Trost. B. M.: Keinan, E. Tetrahedron Lett. 1980, 21, 2595-2598. (c) Trost, B. M.; Pietrusiewicz, K. M. Tetrahedron Leth. 1985, 26 , 4039-4)42. (d) Mendez. M.: Cuerva. T. M.: Gomez-Bengoa. E:: Cardenas, D. J.: Echavarren, A. M. Chem. Eur. J. 2002. 8. $3620-3628$.

8. At the initial stage of this work, the reaction of Baylis-Hillman alcohol 1 a and allyltributylstannane was examined. however. we did not observe the formation of $3 \mathbf{a}$. The reaction of the acetate of 1a was also examined. Although the reaction rate of Baylis-Hillman acetate was faster than that of the bromide, appreciable amounts of side products including $3 \mathrm{a}-Z$ (ca. $5 \%$ ) and secondary adduct (ca. $5 \%$ ) was observed.

9. For the synthesis of cinnamyl bromide derivatives in a stereoselective manner, see: (a) Das, B.; Banerjee, J.; Ravindranath, N. Tetrahedron 2004, 60,8357-8361. (b) Femandes, L.; Bortoluzzi, A. I. Sa. M. M. Tetrahedron 2004. 60.9983-9989. (c) Sa. M. M.: Ramos, M. D: Fenandes. L. Tetrahedion 2006, 62, $11652-11656$. (d) Deng. I.; Hu, X.-P.; Huang. T.-D.; Yu, S.-B.; Wang, D.-Y; Duan, Z.C. Zheng, Z. J. Org. Chent 2008, 73, 2015-2017. (e) Buchholz, R.; Hoffmant, H.; Martin, R. Hell Chm. Acta 1991, 74, 1213-1220 (f) Mazdiyasni, H.: Konopacki, D. B.; Dicliman, D. A.: Zydowsky, T. M. Tetrahedron Lett. 1993, 34, 435438 . (g) Basavaiah, D.; Reddy, K. R.; Kumaragurubaran, N. Nature Protocols 2007, 2, 2665-2676. (h) Lee, K. Y.; Lee, Y. J.; Kim. J. N. Bull. Korean Chem. Soc. 2007, 28, 143-146. (i) Lee, K. Y.; Park. D. Y.: Kim. I. N. Bull. Korean Chem. Soc. 2006. 27. 1489-1492.

10. Treatment of $1 \mathrm{a}$ and $1 \mathrm{~b}$ with $48 \%$ aqueous $\mathrm{HBr}$ at room temperature afforded $2 \mathbf{a}$ and $2 \mathbf{b}$ in 81 and $76 \%$, respectively. The stereochemistry of $2 \mathrm{a}$ and $2 \mathrm{~b}$ was found to be very pure $(Z$-fonm. $>97 \%$ by ${ }^{1} \mathrm{H} N \mathrm{MR}$ ). The reaction of $1 \mathrm{c}$ and $\mathrm{HBr}$ afforded $2 \mathrm{c}$ as a mixture of $E: Z$ isomers $(84 \%, E: Z=4: 1)$. Thus, we first converted $1 \mathrm{c}$ into the corresponding primary alcohol with aqueous $\mathrm{H}_{2} \mathrm{SO}_{4}(63 \%)$, ${ }^{102}$ and synthesized $2 \mathrm{c}$ by treatment with $\mathrm{PBr}_{3}\left(51 \%\right.$, ca. $95 \% E$ by ${ }^{1} \mathrm{H}$ NMR $)$. For the stereoselective synthesis of primary alcohol, see: (a) Basavaiah, D.; Kumaragurubaran, N.; Padmaja, K. Sinlett 1999, 1630-1632.

11. Deng, B.-L.: Lepoivre, J. A.; Lemiere, G. Eur. J. Oig. Chem. $1999.2683-2688$.

12. Murata, M.; Watanabe, S.; Masuda, Y. Synlett 2000, 1043-1045.

13. For the similar cinnamyltin compounds, see: (a) Richter, F.; Weichmann, H. J. Oiganometal Chem. 1994, 466, 77-87. (b) Fouquet, E.: Pereyre, M.: Rodriguez, A. L.; Roulet, T. Bull. Soc. Chim. Fl: 1997, 134, 959-967.

14. (a) Bumett, D. A.; Caplen, M. A.; Domalski, M. S.; Browne, M. E.; Davis, H. R., Jr.; Clader, J. W. Bioorg. Med. Chem. Lett. 2002, 12, $311-314$. (b) Hervet, M.; Thery, I.: Gueiffier, A.; Enguehard-Gueiffier, C. Helv. Chim. Acta 2003, 86, 346l-3469.

15. For the dimerization product 8a, see: Li, T.: Qiarn, W.; Zhang, Y. Tetwhedron 2004, 60, 5793-5798.

16. (a) Thibonnet, I.; Vu, V. A.; Berillon, L: Knochel, P. Tetrahedron 2002, 58, 4787-4799. (b) Thibonnet, T.; Knochel, P. Tetwhedron Lett. 2000, +1, 3319-3322. 